\title{
Short term analysis of total hip replacement done with constrained acetabular component - functional and radiological outcome
}

\author{
Pravin K. Vanchi, Raghav Ravi Veeraraghavan*, Saravanan Vasudevan, \\ Mohan Kumar Murugesan
}

Department of Orthopaedics, Sri Ramachandra Medical College and Research Institute, Chennai, Tamil Nadu, India

Received: 19 December 2021

Revised: 21 December 2021

Accepted: 03 January 2022

\section{*Correspondence:}

Dr. Raghav Ravi Veeraraghavan,

E-mail: raghavravi2193@gmail.com

Copyright: (c) the author(s), publisher and licensee Medip Academy. This is an open-access article distributed under the terms of the Creative Commons Attribution Non-Commercial License, which permits unrestricted non-commercial use, distribution, and reproduction in any medium, provided the original work is properly cited.

\section{ABSTRACT}

Background: Dislocation remains at the forefront of complications after primary total hip arthroplasty (THA). In our study, we talk about the use of constrained liners and its outcomes in an unstable hip.

Methods: The total number of patients included in the study was 15 . The total number of hips in the study were 15 . The age group of the patients varied between 51 years and 89 years with mean age group of 73 years. The most common indication in our study was dislocation contributing $60 \%(n=9)$ of the hips.

Results: The post-operative mean Harris hip score (HHS) at immediate post-operative was 67.6. There was a gradual improvement in the HHS through 6 months (77.6), one year (83.3), two years (86.7) and 3 years (90.33). There were no cases with post-operative septic or aseptic loosening in the radiological analysis. The mean cup inclination was 34.3 . Out of the 13 hips, in eight hips the femoral stem was in varus. Five hips had a centrally placed femoral stem. Stability management in THA have seen the component design take centre stage, primarily with the use of larger diameter femoral heads and the rise in popularity of constrained acetabular liners (CAL). Several authors have done studies which talk about the stability of this implant design and the reliability of this implant in unstable hips and in hips where instability was expected.

Conclusions: In our study, proximal femur tumours, dislocated total hips, abductor insufficiencies and aseptic loosening all showed good results.

Keywords: Total hip arthroplasty, Harris hip score, Constrained acetabular liners

\section{INTRODUCTION}

Dislocation remains at the forefront of complications after primary total hip arthroplasty (THA). The natural history of THA dislocation results in a suboptimal outcome for the patient accompanied by the ongoing risk of recurrent instability and revision surgery. ${ }^{1}$ Instability after revision THA represents an even greater burden and is the leading indication for re- revision surgery. ${ }^{2}$ In the past two decades, stability management in THA have seen the component design take centre stage, primarily with the use of larger diameter femoral heads and the rise in popularity of constrained acetabular liners (CAL). The use of a constrained acetabular liner in total hip replacement has become a popular procedure for patients at high risk of dislocation (intra-operative instability); no abductor function and treatment of recurrent dislocation.

A constrained liner is a device consisting of a liner and reinforcing ring. The polyethylene liner fits into the acetabular metal shell and articulates with the femoral head. The articular surface opening of the constrained liner is reduced slightly to allow for mechanical capture of the femoral head. The reinforcing ring fits into a groove on the outer surface of the liner to reinforce the capture of the femoral head within the liner (dual capturing mechanism). 
This mechanism provides increased stability and reduces the chance of dislocation.

Objective of our study is to use constrained liner for patients requiring a stable hip due to the instability caused due to various factors and to assess its functional and radiological outcome.

\section{METHODS}

A total of 15 patients were included in our study and all patients were having only unilateral hip problems. All patients who required a constrained liner for an unstable hip was included in our study and thus there wasn't a calculated sample size. It was a prospective and a retrospective study and was done in Sri Ramachandra Institute of Higher Education and Research (SRIHER) from March 2018 to July 2021. All patients in whom constrained liner was used in primary and revision total hip arthroplasty were included in study. The complete follow up period was achieved for all the hips which were included. Once included, the complete medical records of the patient was documented along with the indication of the surgery. The pre-operative radiographs (both standard anteroposterior view and lateral views were used). The pre-operative modified Harris hip score was also recorded based on patient's history and case sheet documentation. Post operatively, standard radiographs were taken. The patients were followed up from immediate post-operative period until 3 years. Follow up was done every 6 months and the functional and radiological parameters were recorded. Clinical and radiological analysis were monitored by 3 observers who weren't a part of the index surgical procedure. Modified Harris hip score (HHS) was used for the functional assessment which encompassed all components including pain, mobility, gait and activities. The radiographs taken were used to assess dislocation, loosening, subsidence, acetabular cup inclination, femoral stem position, vertical subsidence, migration of acetabular cup and other complications. The loosening was checked using the Gruen zones (3) for the femoral component and the DeLee-Charnley zones for the acetabular component. The other parameters of the X-ray was done in the PACS with digital measurements. Institutional review board approval was obtained for the study.

\section{Patient cohort and surgical data}

The total number of patients included in the study was 15 . One patient out of this was not followed up after the $6^{\text {th }}$ month as the patient expired due to medical complication unrelated to the surgery. The total number of hips in the study were 15 . The age group of the patients varied between 51 years and 89 years with mean age group of 73 years. There was a comparable sex distribution with $53 \%$ $(\mathrm{n}=8)$ male patients and $47 \%(\mathrm{n}=7)$ female patients. $60 \%$ $(n=9)$ had a left hip pathology and $40 \%(n=9)$ had a right hip pathology (Figure 1). The most common indication in our study was dislocation contributing $60 \%(n=9)$ of the hips (Figure 2). 3 patients had aseptic proximal femoral loosening and 3 others had septic loosening. Among the proximal femur tumours, two were chondrosarcoma and one was leiomyosarcoma. In my study, mean pre-operative HHS (26.41) was not evenly distributed due to the varied indications. Proximal femur tumours might have a near normal HHS and patients with an unstable implant and impending dislocation might have a poor HHS.

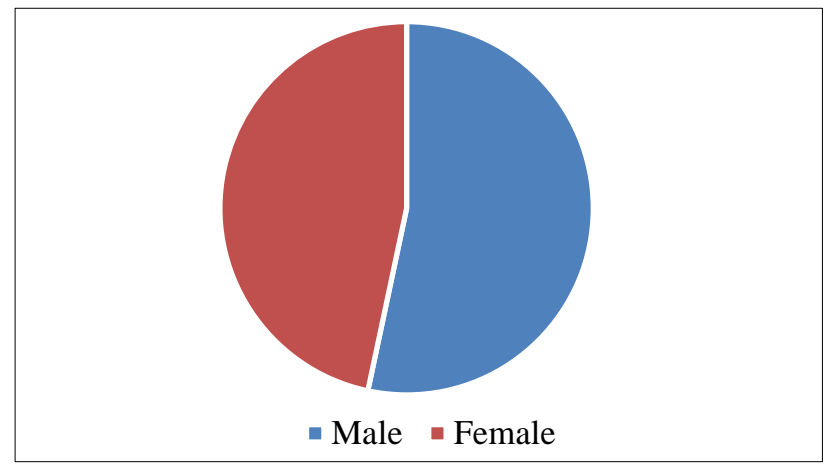

Figure 1: Sex distribution.

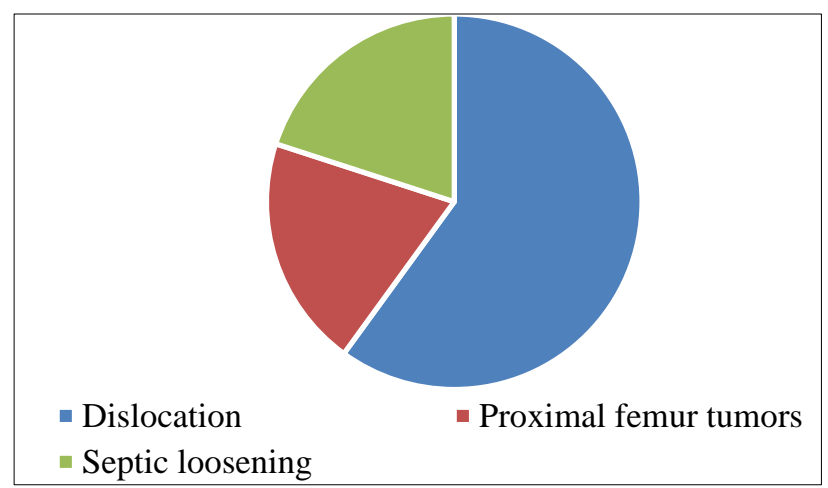

Figure 2: Indications.

It was a descriptional study and statistical analysis was done based on Pearson's Chi-square tests.

All the procedures were done by a single surgeon via the posterior approach. An abduction pillow was used for initial post-operative period. The patients were made to partially weight bear initially with the help if a walker and was then mobilised with a stick for another 6 weeks with bearing full weight.

\section{RESULTS}

The post-operative mean HHS at immediate post-operative was 67.6. There was a gradual improvement in the HHS through 6 months (77.6), one year (83.3), two years (86.7) and 3 years (90.33) (Figure 3 ).

There were no cases with post-operative septic or aseptic loosening in the radiological analysis. The mean cup inclination was 34.3. Out of the 13 hips, in eight hips the femoral stem was in varus. Five hips had a centrally placed femoral stem. There were no cases with migration of the 
acetabular cup in our series. In one case we had an incidence of vertical subsidence. The patient had a limb length shortening of $3 \mathrm{~cm}$.

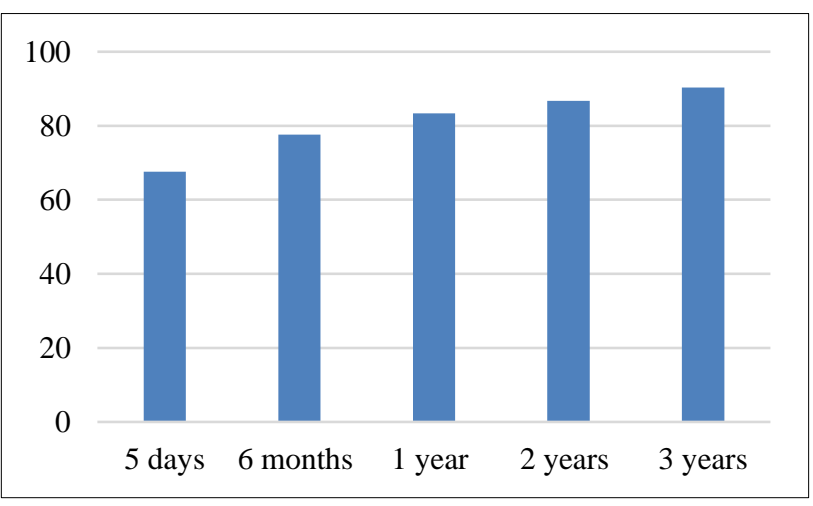

Figure 3: Harris hip score.

In our study we had both constrained liner related complications and complications which weren't related to the liner. In a patient we had a complication of locking ring failure. The patient expired following this and hence follow up was lost. We also had a case of dislocation due to head and neck dissociation. There was one another patient, a 70 year old female for whom we were revising a dislocated hemiarthroplasty with a constrained hip. While trying to remove the stem, there was a periprosthetic fracture of the femur shaft which was then treated with cable and plate construct after revising the hip.

\section{DISCUSSION}

Instability after revision THA represents an even greater burden as it requires more soft tissue insult and increases the chances of instability and is the leading indication for re- revision surgery. ${ }^{3}$ Stability management in THA have seen the component design take centre stage, primarily with the use of larger diameter femoral heads and the rise in popularity of constrained acetabular liners (CAL). The use of a constrained acetabular has provided the surgeon a great fall back option even for unexpected intra-operative situations like instability. Our study talks about this particular implant design and measures its performance both functionally and radiologically.

Several authors have done studies which talk about the stability of this implant design and the reliability of this implant in unstable hips and in hips where instability was expected. Anderson et al did a study where the followed up hips with constrained liner for a study period of 33 months. ${ }^{4}$ They had a patient base of 32 samples (Figure 4). Their study's HHS post-operatively was 81 which is a fairly good outcome. The complication (constrained liner related) rate was higher in their study $(n=4)$ when compared with my study $(n=2)$. In my study the complications were locking ring failure due to patient factor (violence at home) and head and neck dissociation. The four cases in their study were all dislocations post operatively due to combined patient and procedural factors. But this difference wasn't considered to be significant as the sample size was more in their study.

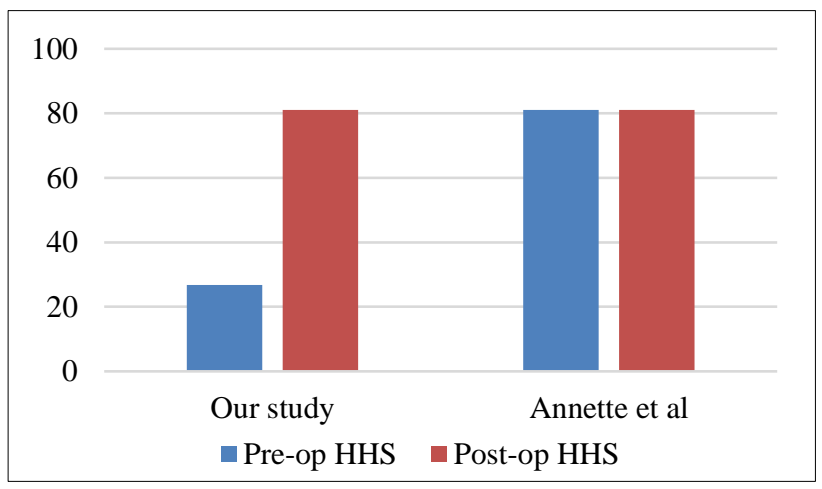

Figure 4: Comparative analysis with Annette et al.

Rady et al did a study with 15 patients with patients who had recurrent hip dislocations and soft tissue fibrosis for which the revision procedure was done. ${ }^{5}$ In their study, the post-operative HHS was 88 which is significantly higher when compared with our study (81.06). They had only one complication which was not component related.

The major setback in our study was that we did not have a long term follow up of our patients. We did not have the complication rate which might usually arise as a long term issue for patients operated with a constrained liner. Siavashi et al did a similar study as ours but had a follow up of 5.5 years. $^{6}$ The complications that arose in their patients were failure of the implant and deep seated infection apart from the ones we experienced in our study.

The use of the constrained liner is a major advent when it comes to tumour resection procedures as it involves severe soft tissue dissection and resection. The main reason for soft tissue resection apart from the usual dissection is to achieve a good tumour margin so that the resection has an adequate clearance. Jawad et al did a study exclusively for proximal femur tumour as an indication. ${ }^{7}$

In my study proximal femur tumour resection was done by posterior approach. Reconstructive options for the proximal femur after massive bone loss include THA with a large head and constrained acetabular liner. After the proximal femur is resected, the hip abductors can be anchored down to the prosthesis. However, despite such reconstructions, significant loss of abduction strength occurs, commonly characterized clinically by a Trendelenburg gait. Consequently, when the abductor force is diminished, stability of the hip is compromised and dislocation may ensue. If the capsule can be preserved and purse-stringed around the implant, stability is often not a concern despite compromised hip abductors.

After proximal femoral resection, reaming and placement of the acetabular component is neutral to the anatomy of the patient's acetabulum. No additional version is used, and the intraoperative acetabular positioning is 
significantly less (approximately $15^{\circ}$ ) than the standard placement of the acetabulum performed in THA. After the acetabulum has been reamed, the cup is placed anatomically so the overlap of the cup to the acetabular rim is symmetric. No change from the patient's anatomic version is put into the cup, regardless of structural or morphologic changes of the acetabulum. In most cases, the acetabular cup is placed at approximately $15^{\circ}$ to $20^{\circ}$ of anteversion, which is the mean acetabular version when evaluating the upper- and mid-level version. A mean acetabular version of $15^{\circ}$ to $20^{\circ}$ is supported by Perreira et al. $^{8}$

The whole methodology used was the same as our study. This meticulous planning and component placement ensured stability of the prosthesis. The use of a constrained liner on top of this increased the stability and reduced the chance of dislocation.

Radical resection of proximal femoral tumours, the defect is reconstructed with long reef and solution stem, as we said before the abductors has been tied to the prosthesis.

The study period was 30.4 months and the sample size were 33 patients (male and female ratio is 17:16). Of the 33 patients, 17 were alive and 16 had died. Reason for using the constrained liner in this study is: bone metastasis recalcitrant to treatment/impending pathologic fracture; salvage procedure for recurrence or failure of primary implant; and primary malignancy.

In my study there were three tumour cases out of which, two cases were proximal femur chondrosarcoma stage $2 \mathrm{~B}$ and one case was proximal femur leiomyosarcoma. Preoperative biopsy done which confirmed the diagnosis of proximal femur chondrosarcoma and leiomyosarcoma.

But in our study all 3 cases are primary malignancy, radical resection of proximal femoral tumours with abductors and reconstructed the abductors with mesh arthroplasty which is more prone for dislocation hence constrained liner used. In our study 3 patients they excellent functional outcome (postop HHS-92.9) in the follow up of 3 years.

We had complications in our study which were both related and unrelated to the constrained liner. In a patient we had a complication of locking ring failure. The patient had presented to us with a dislocated hemiarthroplasty implant (Figure 5). We planned and took him up for a revision surgery. Intraoperatively, we found that the version of the femoral stem was found to be normal. There was an abductor insufficiency and there was no soft tissue tension hence, we planned to use a constrained acetabular liner. Two months post procedure, patient had an incident of slip and fall at his residence. We then planned for a closed reduction and relocation of the prosthesis. Postoperatively, the patient's HHS was 65 after 6 months. The patient expired following this and hence follow up was lost. A complication related to the stem arose when a two stage revision surgery was done for a patient with an infected implant. During the second stage of the revision which was the definitive surgery, we used a constrained liner as the choice of the prosthesis. Six months following the surgery the patient presented with gross limb length discrepancy of $3 \mathrm{~cm}$. X-ray showed vertical subsidence of femoral stem (Figure 6).

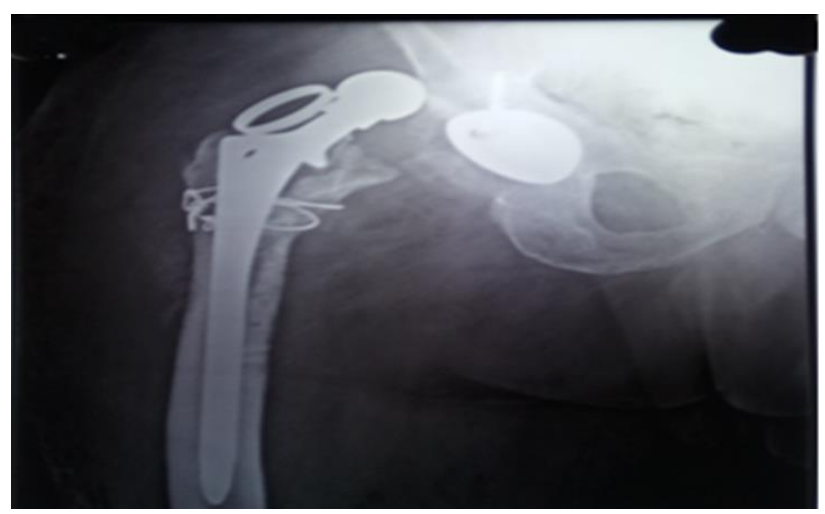

Figure 5: Locking ring failure.

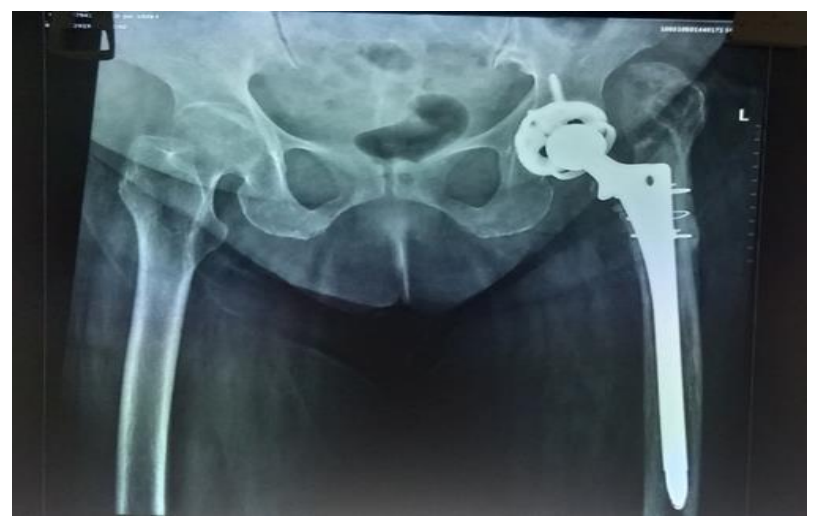

Figure 6: Vertical subsidence of femoral one year post implantation.

There are a few limitations in this study, the sample size being less is one. This is mainly attributed to the rarity of usage of such implants in a regular basis. The follow up is also a short term follow up. Probably a long term follow up would give a better perspective regarding this implant's performance.

\section{CONCLUSION}

In our study, proximal femur tumours, dislocated total hips, abductor insufficiencies and aseptic loosening all showed good results. Our mean HHS pre-operatively was26.7 (5-90) which significantly increased to 81.06 (67.6090.33 ) post operatively. Short term analysis showed good outcome, however long term follow- up is necessary. Patients should be instructed that significant reduction in the range of motion is inherent to the design characteristic of a constrained acetabular liner, and activities that may force the joint to exceed those range of motion limits should be avoided constrained liner done for revision total hip arthroplasty without optimizing other aspects of the 
reconstruction leads to a high rate of recurrent failure. There are wide range of unstable hip solutions for which constrained liner can be a reliable implant of choice.

Funding: No funding sources

Conflict of interest: None declared

Ethical approval: The study was approved by the institutional ethics committee

\section{REFERENCES}

1. Kotwal RS, Ganapathi M, John A, Maheson M, Jones $\mathrm{S}$. Outcome of treatment for dislocation after primary total hip replacement. JBJS (Br). 2009;91-B:321-6.

2. Bozic KJ, Kurtz SM, Lau E, Ong K, Vail TP, Berry DJ. The Epidemiology of revision total hip arthroplasty in the United States. JBJS (Am). 2009;91:128-33.

3. Engh CA, Massin P, Suthers KE. Roentgenographic assessment of the biologic fixation of poroussurfaced femoral components. Clin Orthop Relat Res. 1990;257:107.

4. Andersen AV, Kjersgaard AG, Solgaard S. Trilogyconstrained acetabular component for recurrent dislocation. ISRN Orthop. 2013;629201.
5. Rady AE, Asal MK, Bassiony AA. The use of a constrained cementless acetabular component for instability in total hip replacement. Hip Int. 2010;20(4):434-9.

6. Siavashi B, Pendar E, Golbakhsh MR, Savadkoohi DG, Zehtab MJ, Sadat MM. Constrained Liner in Total Hip Arthroplasty. J Chem Pharm Res. 2016;8(10):112-6.

7. Jawad MU, Brien EW. Proximal femoral reconstruction with a constrained acetabulum in oncologic patients. Orthopedics. 2014;37(2):187-93.

8. Perreira AC, Hunter JC, Laird T, Jamali AA. Multilevel measurement of acetabular version using 3-D CT-generated models: implications for hip preservation surgery. Clin Orthop Relat Res. 2011;469(2):552-61.

Cite this article as: Vanchi PK Veeraraghavan RR, Vasudevan S, Murugesan MK. Short term analysis of total hip replacement done with constrained acetabular component - functional and radiological outcome. Int J Res Orthop 2022;8:119-23. 